\title{
Propriedade antifúngica do extrato aquoso de Allium sativum frente a fungos isolados
}

\section{do solo}

\author{
Antifungal property of the aqueous extract of Allium sativum against fungi isolated from the soil \\ Propiedad antifúngica del extracto acuoso de Allium sativum frente a hongos aislados del suelo
}

Recebido: 02/12/2020 | Revisado: 17/12/2020 | Aceito: 27/01/2021 | Publicado: 02/02/2021

José Alisson da Silva Lima

ORCID: https://orcid.org/0000-0002-5021-0896 Centro Universitário Tiradentes, Brasil

E-mail: alissonlima_2@hotmail.com

Lais Nicolly Ribeiro da Silva

ORCID: https://orcid.org/0000-0003-4300-838X Universidade Federal de Alagoas, Brasil

E-mail: laisnicollyribeiro@gmail.com

Rodrigo José Nunes Calumby

ORCID: https://orcid.org/0000-0002-2313-5552

Universidade Federal de Alagoas, Brasil

E-mail: rjnc_biomed@hotmail.com

Jayane Omena de Oliveira

ORCID: https://orcid.org/0000-0003-2830-9379

Universidade Federal de Alagoas, Brasil

E-mail: jayaneomena@gmail.com

Davi Porfirio da Silva

ORCID: https://orcid.org/0000-0002-1856-4512 Universidade Federal de Alagoas, Brasil E-mail: daviporfirio14@hotmail.com

Valter Alvino

ORCID: https://orcid.org/0000-0001-7132-3622 Universidade Federal de Alagoas, Brasil

E-mail: valteralvinos@hotmail.com

Rossana Teotônio de Farias Moreira

ORCID: https://orcid.org/0000-0002-0881-1997 Universidade Federal de Alagoas, Brasil E-mail: rossanateo@hotmail.com

Maria Anilda dos Santos Araújo

ORCID: https://orcid.org/0000-0002-9833-4265 Centro Universitário Tiradentes, Brasil E-mail: fungosanilda@gmail.com

\section{Resumo}

Allium sativum L. é um condimento de sabor característico e composição fitoquímica rica em princípios ativos com atividade antimicrobiana. Este estudo teve por objetivo avaliar o potencial antifúngico in vitro do extrato aquoso de A. sativum frente à fungos patogênicos isolados do solo. Foram coletadas 30 amostras do solo em três praças públicas da cidade de Maceió - Al e, em seguida, processadas para o isolamento de fungos por meio da técnica de suspensão de solo. Os fungos foram identificados por meio da visualização dos aspectos macroscópicos e microscópicos das colônias. O extrato aquoso de A. sativum (EA) foi obtido por meio de infusão do bulbo fragmentado e os testes de suscetibilidade antifúngica foram realizados através de difusão em ágar. Como resultados, foram obtidas 256 Unidades Formadoras de Colônias (UFC), das quais 110 (42,9\%) correspondiam a Aspergillus sp., 52 (20,3\%) a Mycelia sterilia, 28 (10,9\%) a Penicillium sp. e 24 (9,4\%) a Beauveria bassiana. Em relação ao EA, este demonstrou atividade antifúngica contra Beauveria bassiana, Aspergillus sp. e Penicillium sp., exibindo halos de inibição que variaram de 10 a $30 \mathrm{~mm}$. Esses achados demonstram a atividade antifúngica do alho contra diferentes fungos oriundos do solo, estando esta relacionada a sua rica composição fitoquímica que proporciona ação fungicida direta e atua na inibição do micélio e na germinação dos esporos.

Palavras-chave: Allium sativum; Propriedades antifúngicas; Solo.

\begin{abstract}
Allium sativum L. is a flavoring with a characteristic flavor and phytochemical composition rich in active ingredients with antimicrobial activity. This study aimed to evaluate the antifungal potential in vitro of the aqueous extract of $A$. sativum against pathogenic fungi isolated from the soil. Thirty soil samples were collected in three public squares in the city of Maceió - Al and then processed for the isolation of fungi using the soil suspension technique. Fungi were identified by visualizing the macroscopic and microscopic aspects of the colonies. The aqueous extract of A. sativum (AE) was obtained through infusion of the fragmented
\end{abstract}


bulb and the antifungal susceptibility tests were performed through agar diffusion. As a result, 256 Colony Forming Units (UFC) were obtained, of which 110 (42.9\%) corresponded to Aspergillus sp., 52 (20.3\%) Mycelia sterilia, 28 (10.9\%) Penicillium sp. and $24(9.4 \%)$ Beauveria bassiana. In relation to AE, it showed antifungal activity against Beauveria bassiana, Aspergillus sp. and Penicillium sp., exhibiting inhibition halos that varied from 10 to $30 \mathrm{~mm}$. These findings demonstrate the antifungal activity of garlic against different fungi from the soil, which is related to its rich phytochemical composition that provides direct fungicidal action and acts in the inhibition of the mycelium and in the germination of the spores.

Keywords: Allium sativum; Antifungal properties; Ground.

\section{Resumen}

Allium sativum L. es un aromatizante de sabor característico y composición fitoquímica rica en principios activos con actividad antimicrobiana. Este estudio tuvo como objetivo evaluar el potencial antifúngico in vitro del extracto acuoso de A. sativum frente a hongos patógenos aislados del suelo. Se recolectaron treinta muestras de suelo en tres plazas públicas de la ciudad de Maceió - Al, y luego se procesaron para aislar hongos mediante la técnica de suspensión de suelo. Los hongos se identificaron visualizando los aspectos macroscópicos y microscópicos de las colonias. El extracto acuoso de A. sativum (EA) se obtuvo mediante infusión del bulbo fragmentado y las pruebas de susceptibilidad antifúngica se realizaron mediante difusión en agar. Como resultado se obtuvieron 256 Unidades Formadoras de Colonias (UFC), de las cuales 110 (42,9\%) correspondieron a Aspergillus sp., 52 (20,3\%) a Mycelia sterilia, 28 (10,9\%) a Penicillium sp. y 24 (9,4\%) a Beauveria bassiana. En relación al EA, éste mostró actividad antifúngica contra Beauveria bassiana, Aspergillus sp. y Penicillium sp., exhibiendo halos de inhibición que variaron de 10 a $30 \mathrm{~mm}$. Estos hallazgos demuestran la actividad antifúngica del ajo frente a diferentes hongos del suelo, lo que se relaciona con su rica composición fitoquímica que le brinda acción fungicida directa y actúa en la inhibición del micelio y en la germinación de las esporas.

Palabras clave: Allium sativum; Propiedades antifúngicas; Suelo.

\section{Introdução}

O alho (Allium sativum L.) consiste em uma planta herbácea, pertencente à família Liliaceae, cujo bulbo é composto por folhas lineares e escamiformes, amplamente utilizada como planta aromática e condimentar. No entanto, além de ser uma ótima especiaria alimentar, este vegetal apresenta diversos princípios ativos microbicidas que podem desempenhar um importante papel na defesa contra diferentes fitopatógenos e enfermidades humanas (Leite et al., 2012; Fontenele et al., 2015; Caldas et al., 2019; Candido et al., 2019).

A. sativum apresenta em sua composição fitoquímica mais de 100 compostos biologicamente ativos, destacando-se o selênio, o ajoeno, o tiosulfinato e a alicina, óleo responsável pelo seu odor característico. A Alicina, também conhecida como dialiltiosulfinato, é obtida através da trituração do vegetal com auxílio da enzima Alinase e, além do seu forte aroma, este fitoquímico é considerado o princípio ativo de maior valor biológico do extrato de alho, devido às suas atividades antimicrobiana, antitrombótica, hipolipidêmica e anticancerígena (Caldas et al., 2019; Silva, Santos \& Siqueira, 2020).

O solo é o habitat de diversas espécies de microrganismos, dentre eles os fungos, participantes ativos do processo de biodegradação, ciclagem de nutrientes e consequentemente manutenção do ecossistema. Porém, por mais que estes microrganismos proporcionem vários benefícios ao solo, também são causadores de inúmeras infecções fúngicas oportunistas, como as onicomicoses, sendo as praças e parques locais propícios para a contaminação, uma vez que há um contato direto e intenso entre crianças e animais com a areia (Takahashi et al., 2011).

As doenças tropicais, cuja proliferação é intensificada em regiões de clima quente e úmido, apresentam maior incidência em países localizados nas proximidades da linha do Equador e entre os trópicos de Câncer e Capricórnio, exatamente onde se localiza a maior parte do território brasileiro. Maceió, capital do estado de Alagoas, é caracterizada como sendo uma cidade tipicamente de clima tropical, apresentando doenças infecciosas e parasitárias denominadas de patologias geográficas tropicais (Sobral; Miranda; Mascarenha \& Silva, 2018)

Considerando a rica composição fitoquímica, as atividades antifúngicas sobre diversos fitopatógenos e as inúmeras propriedades antimicrobianas apresentadas pelo alho, este estudo teve por objetivo avaliar o potencial antifúngico in vitro do extrato aquoso de A. sativum no combate a fungos patogênicos isolados em solos de praças públicas da cidade de Maceió, Alagoas. 


\section{Metodologia}

\subsection{Coleta e processamento das amostras}

As coletas de amostras do solo foram realizadas em praças públicas da cidade de Maceió, sendo elas: praça Sinimbú (940'04.9"S 3544'01.3"W), praça Dom Pedro II (9³9'56.4"S 3544'10.1"W) e praça Centenário (9³9'07.7"S 3544'00.4"W). Para a realização das coletas foram utilizadas espátulas e coletores universais estéreis, que auxiliaram na raspagem do solo e acondicionamento. Ao todo, foram coletadas 30 amostras do solo (cerca de $10 \mathrm{~g}$ ), sendo 10 amostras em cada área de recreação, a coleta foi realizada em pontos equidistantes, com uma distância aproximada de 15 metros entre uma e outra (Oliveira et al., 2020).

Foram estabelecidos alguns critérios de identificação das amostras utilizando-se os códigos "S" para solo superficial e “P” para solo profundo. As amostras de solo profundo foram obtidas através da extração em uma depressão de $20 \mathrm{~cm}$ (Lacaz et al., 2002). Ao final das coletas, as amostras foram acondicionadas em caixas térmicas e transportadas para o laboratório de Microbiologia do Centro Universitário Tiradentes (UNIT).

Em balança analítica foi pesado um total de 1,0 g de cada amostra e colocada em tubos de ensaio. Em seguida, adicionouse $20 \mathrm{~mL}$ de solução salina para a homogeneização da suspensão em vórtex por seis minutos. Transcorrido o tempo, as soluções ficaram em repouso em tubos fechados em uma bancada limpa previamente com álcool a 70\%, por um período máximo de 40 minutos para sedimentação dos resíduos permitindo assim a flutuação das estruturas fúngicas, como hifas e esporos. Posteriormente, com auxílio de uma pipeta semiautomática de $1000 \mu 1$, realizou-se diluição seriada das suspensões $\left(10^{-1}\right.$ a $\left.10^{-3}\right)$, e desta última diluição, foi realizado semeio de $100 \mu \mathrm{L}$ do sobrenadante através de espalhamento radial em placas de Petri contendo ágar Sabouraud Dextrose acrescido de cloranfenicol. As placas foram semeadas em duplicata, incubadas à temperatura ambiente $\left(25^{\circ}-30^{\circ} \mathrm{C}\right)$ e observadas até o $20^{\circ}$ dia de incubação (Zaitz et al., 2010).

\subsection{Identificação dos fungos}

Após constatação de crescimento fúngico foi realizada contagem das colônias obtidas com auxílio de um contador de colônias digital (LS 6010, LAB1000), sendo estabelecidos critérios de divisão dos fungos isolados para cada amostra coletada referente às características do micélio: cor, textura, aspecto e borda das colônias.

A identificação dos fungos baseou-se na associação de aspectos macroscópicos e microscópicas do exame direto da cultura, sendo os fungos filamentosos confirmados pela estimulação da esporulação pela técnica de microcultivo em lâmina utilizando-se ágar Lactrimel (Hoog et al., 2000; Lacaz et al., 2002; Sidrim \& Rocha, 2004; Zaitz et al., 2010; Calumby et al., 2019). Após identificação, foram preparadas suspensões em microtubos Eppendorf® com água destilada estéril para conservação dos isolados à $4^{\circ} \mathrm{C}$ (Sidrim \& Rocha, 2004).

\subsection{Obtenção do extrato aquoso de $A$. sativum}

Para obtenção do extrato bruto foi utilizada técnica descrita por Lubian et al. (2010), cujo preparo consistiu na utilização de $20 \mathrm{~g}$ do bulbo de Allium sativum fragmentado para $1000 \mathrm{~mL}$ de água destilada, sendo realizadas cinco etapas de preparação para obtenção do extrato aquoso estéril. Inicialmente, $20 \mathrm{~g}$ de matéria vegetal foram suspensas em $300 \mathrm{~mL}$ de água destilada a $100^{\circ} \mathrm{C}$, permanecendo em infusão por $24 \mathrm{~h}$, em local devidamente fechado e protegido da luz. Após este período, realizou-se filtragem do fluido e foram adicionados mais $350 \mathrm{~mL}$ de água destilada ao resíduo vegetal da filtração à temperatura de $100^{\circ} \mathrm{C}$, sendo este mantido em infusão por mais $24 \mathrm{~h}$ de forma semelhante à primeira fase. A extração obtida nesta segunda etapa foi novamente filtrada e, ao resíduo resultante, foram adicionados mais $350 \mathrm{~mL}$ de água destilada a $100^{\circ} \mathrm{C}$ e mantidos em infusão por mais $24 \mathrm{~h}$, concluindo o processo de extração. Ao final da última filtragem foi obtido o extrato aquoso bruto. Este foi submetido à ebulição por mais cinco minutos e, em seguida, esterilizado por filtração através de membrana Milipore® de 0,22 
$\mu \mathrm{m}$. Por fim, esta solução foi acondicionada em tubos de ensaio estéreis, mantidos a $4^{\circ} \mathrm{C}$ e protegidos da luz.

\subsection{Atividade antifúngica de $A$. sativum}

Para avaliação da suscetibilidade antifúngica foi utilizado método de difusão em ágar, conforme o Clinical and Laboratory Standards Institute - CLSI (2018). O extrato de Allium sativum foi testado sobre os isolados fúngicos identificados no solo, sendo eles: Absidia sp., Acremonium sp., Aspergillus sp., Aspergillus niger, Beauveria bassiana, Penicillium sp. e Mycelia sterilia.

Os inóculos foram preparados em tubos contendo solução salina tamponada estéril e tween 20 (2\%) para auxiliar na solubilização dos esporos. As suspensões fúngicas foram determinadas pela transmitância equivalente à de uma solução-padrão da escala de McFarland 0,5, correspondente a aproximadamente 1,5 x $10^{6}$ células por mL, conforme documento do CLSI (2018). Cada suspensão foi distribuída uniformemente com o auxílio de swabs na superfície de uma placa de Petri contendo ASD. Posteriormente, foram realizados orifícios de $6 \mathrm{~mm}$ de diâmetro com o auxílio de um cilindro para a perfuração de poços, onde foram aplicados $50 \mu \mathrm{L}$ do extrato aquoso a $5 \%$. Como controle negativo foi usado uma solução de DMSO a $2 \%$. As placas foram incubadas a temperatura ambiente por 7 dias e o procedimento foi realizado em duplicata.

Após o período de incubação, as placas foram analisadas quanto à formação de halos de inibição de crescimento. A atividade antifúngica foi considerada quando em pelo menos duas placas foi possível observar a presença de halos de $8 \mathrm{~mm}$ ou mais.

\section{Resultados}

Das 30 amostras avaliadas, foram obtidas 256 Unidades Formadoras de Colônias (UFC), sendo 142 (55,5\%) provenientes do solo profundo e 114 (44,5\%) do solo de superfície. A maior ocorrência de fungos no solo de superfície foi encontrada na praça Sinimbú (86 UFC), enquanto em solo profundo observou-se maior prevalência na praça Centenário (98 UFC). Com relação aos fungos identificados, verificou-se predomínio de fungos filamentosos, destacando-se Aspergillus sp. com 110 UFC (44,5\%), seguido por Mycelia sterilia com 42 UFC (16,4\%) e Penicillium sp. com 34 UFC (13,3\%), conforme Tabela 1. 
Tabela 1 - Ocorrência de UFC de fungos isolados em solo superficial e profundo de praças públicas da cidade de Maceió, Alagoas.

\begin{tabular}{|c|c|c|c|c|c|c|c|c|c|}
\hline \multirow{2}{*}{ Fungos } & \multicolumn{4}{|c|}{ Solo Superficial } & \multicolumn{4}{|c|}{ Solo Profundo } & \multirow{2}{*}{ Número Total de UFC } \\
\hline & PS & PD & PC & Total & PS & PD & PC & Total & \\
\hline Absidia sp. & - & 4 & - & $4(3,5 \%)$ & - & 2 & 2 & $4(2,8 \%)$ & $8(3,1 \%)$ \\
\hline Acremonium sp. & - & 4 & - & $4(3,5 \%)$ & - & 2 & 4 & $6(4,2 \%)$ & $10(3,9 \%)$ \\
\hline Aspergillus niger & 4 & 2 & 12 & $18(15,8 \%)$ & - & - & 6 & $6(4,2 \%)$ & $24(9,4 \%)$ \\
\hline Beauveria bassiana & 24 & - & - & $24(21,0 \%)$ & - & - & - & - & $24(9,4 \%)$ \\
\hline Penicillium sp. & 18 & - & - & $18(15,8 \%)$ & 2 & - & 14 & $16(11,3 \%)$ & $34(13,3 \%)$ \\
\hline Mycelia sterilia & 18 & - & - & $18(15,8 \%)$ & 24 & - & - & $24(17,0 \%)$ & $42(16,4 \%)$ \\
\hline Aspergillus sp. & 22 & 4 & 2 & $28(24,6 \%)$ & 10 & 4 & 72 & $86(60,5 \%)$ & $114(44,5 \%)$ \\
\hline Total & 86 & 14 & 14 & $114(44,5 \%)$ & 36 & 8 & 98 & $142(55,5 \%)$ & $256(100,0 \%)$ \\
\hline
\end{tabular}

Legendas: PS= Praça Sinimbú; PD= Praça Dom Pedro II; PC = Praça Centenário. Fonte: Autores.

No que se refere a atividade antifúngica, o EA de A. sativum apresentou ação inibitória frente a Beauveria bassiana, Aspergillus sp. e Penicillium sp., exibindo halos de $30 \mathrm{~mm}, 15 \mathrm{~mm}$ e $10 \mathrm{~mm}$, respectivamente (Figura 1), evidenciando a importância da pesquisa em proporcionar a busca por novos agentes terapêuticos, que assim como o A. sativum também apresentem propriedade antifúngicas, para que existam novas alternativas para o surgimento de possíveis fungos mais resistentes aos antifúngicos comercializados.

Figura 1. Atividade antifúngica do extrato aquoso de Allium sativum frente a fungos isolados do solo de praças públicas de Maceió, Alagoas.

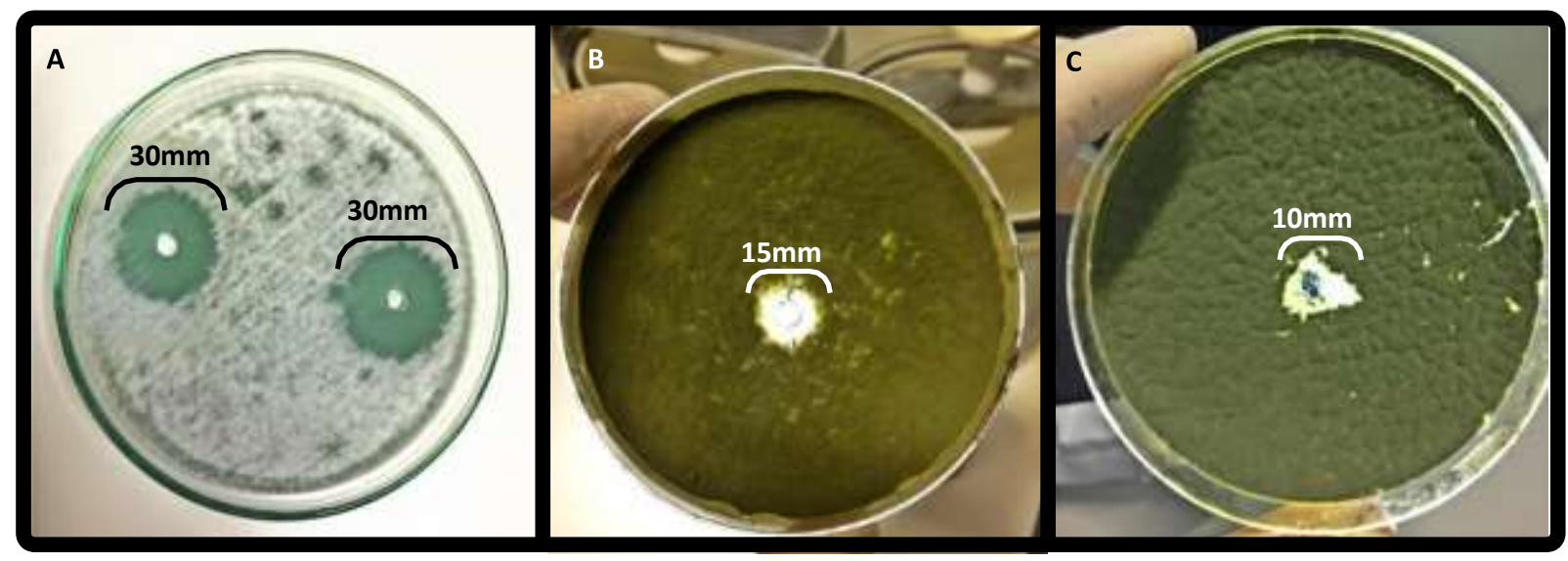

Legenda: A = Beauveria bassiana $; \mathrm{B}=$ Aspergillus sp. e $\mathrm{C}=$ Penicillium $\mathrm{sp}$.

Fonte: Autores.

\section{Discussão}

O solo sob o ponto de vista microbiológico apresenta condições de umidade e temperatura adequadas para a manutenção da vida de diversos microrganismos, especialmente os fungos filamentosos, pois vivem às custas da decomposição da matéria 
orgânica. Estes por sua vez, estão sendo cada vez mais descritos como patógenos causadores de micoses cutâneas (Fontenele et al., 2015; Takahashi et al., 2011).

Pereira et al. (2010) relataram que o solo superficial está constantemente passando por modificações decorrentes de fatores externos e climáticos, alterando a colonização natural da sua microbiota, principalmente em decorrência do vento, pois este age como importante condutor fragmentando as hifas e espalhando os propágulos, enquanto que em amostras do solo profundo estas modificações ocorrem com maior dificuldade, pois o ambiente recebe menor impacto dos fatores externos, o que o torna mais propício para a proliferação de diversos tipos de microrganismos, justificando a maior incidência de fungos provenientes destas amostras nesta pesquisa (Costa et al., 2017).

Nesse seguimento, Takahashi et al. (2011) realizaram levantamento de fungos queratinofílicos em solos de parques e praças públicas no município de São Bernardo do Campo e verificaram maior ocorrência de Penicillium spp., Cunninghamella spp., Fusarium spp. e Acremonium spp., diferente dos achados deste estudo. A maioria dos fungos encontrados eram queratinofílicos, sendo estes constantemente associados a infecções oportunistas como as dermatofitoses, onicomicoses e as otomicoses.

A proposta da utilização dos extratos vegetais configura uma alternativa viável e ecologicamente correta, pois contribui com a preservação do solo e a redução da quantidade de agrotóxicos nos alimentos (Candido et al., 2019). Pesquisas realizadas com o extrato de alho tem indicado o seu potencial no controle de fungos patogênicos e não patogênicos, por este possuir compostos secundários que tanto podem ter ação fungicida direta, quanto na inibição de micélio e na germinação dos esporos (Leite et al., 2012; Brito; Nascimento, 2015; Costa et al., 2017).

O EA tem indicado alto potencial no controle de fungos, especialmente na inibição do crescimento de Aspergillus niger, Penicilium spp. e Fusarium oxysporum, fungos responsáveis por muitas infecções de início insidioso (Costa et al., 2017).

Conforme observado em alguns estudos in vitro, o EA apresenta atividade antibacteriana associada à alicina, uma vez que atua na inibição e destruição de bactérias gram-negativas (Caldas et al., 2019). Por conseguinte, sua utilização também resulta em alterações morfológicas nas hifas quando usada nas espécies: Pythium ultimum, Rhizoctonia solani, Colletotrichum lindemuthianum e Fusarium solani (Costa et al., 2017).

Costa et al. (2017) verificaram que a atividade antimicrobiana in vitro do EA de A. sativum teve início a partir da concentração de $40 \%$ frente a Fusarium subglutinans, inibindo a germinação de esporos. Em outro estudo, Santos et al. (2010) demonstraram que a utilização do extrato de bulbilhos de alho inibiu significativamente o crescimento micelial de Aspergillus niger na concentração de $50000 \mathrm{mg} \mathrm{L}^{-1}(5 \%)$, diferente do observado neste estudo, onde o EA não apresentou atividade antifúngica sobre as cepas de A. niger isoladas. De acordo com Brito e Nascimento (2015), esta inibição interfere no desenvolvimento do fungo e ocorre de forma crescente e proporcional ao aumento das concentrações do extrato.

Os resultados encontrados nesta presente pesquisa convergem com os presentes na literatura, pois mediante a realização dos testes de susceptibilidade, o extrato aquoso de A. sativum apresentou ação inibitória frente a Beauveria bassiana, Aspergillus sp. e Penicillium sp.

\section{Conclusão}

Os resultados evidenciam uma predominância de fungos filamentosos no solo das praças públicas, destacando-se Aspergillus sp., Mycelia sterilia e Penicillium sp. Além disso, após ensaio de susceptibilidade foi possível concluir que o extrato aquoso de A. sativum apresentou atividade antifúngica contra diversos fungos, tais como B. bassiana, Aspergillus sp. e Penicillium sp., atuando na inibição do crescimento desses microrganismos, mediante sua ação fungicida direta e na inibição da germinação dos esporos e do micélio. Diante desses achados, observa-se que as propriedades antifúngicas presentes no alho apresentam-se relevantes, podendo ser utilizadas como alternativa terapêutica. 
No entanto, ainda que esta pesquisa tenha apresentado resultados positivos, faz-se necessário a realização de mais estudos que investiguem a propriedade antifúngica contra espécies de diversos gêneros fúngicos.

\section{Referências}

Bauer, A., Kirby, W., Sherris, J., \& Turck, M. (1966). Antibiotic susceptibility testing by a standardized single disk method. American Journal of Clinical Pathology, 45(4), 493-496.

Brito, N. M. \& Nascimento, L.C. (2015). Potencial fungitóxico de extratos vegetais sobre Curvularia eragrostidis (P. Henn.) Meyer in vitro. Revista Brasileira de Plantas Medicinais, 17 (2), 230-238.

Calumby, R. J. N., Silva, J. A., Silva, D. P., Moreira, R. T. F., Araújo, M. A. S., Almeida, L. M., Grillo, L. A. M., \& Alvino, V. (2019). Isolamento e identificação da microbiota fúngica anemófila em Unidade de Terapia Intensiva. Brazilian Journal of Development, 5(10), 19708-19722.

Candido, J. B. S., Cavalcante, L. V., Peixinho, G. D. S., Silva, G. D. N., Lima, M. O. D., Oliveira, Y. D. M. D., Abreu, L. A. D., \& Amorim, E. P. D. R. (2019). Subprodutos de Allium sativum na inibição do crescimento micelial de Alternaria alternata em tomate (Solanum lycopersicum). Revista Craibeiras de Agroecologia, 4, e9627.

CLSI - Clinical and Laboratory Standards Institute. Method for antifungal disk diffusion susceptibility testing of yeasts: approved guideline M44-A2. CLSI, Wayne, PA, USA, 2018.

Costa, N. C., Chagas Junior, A. F., Ramos, A. C. C., Soares, L. P., \& Scheidt, G. N. (2017). Revista Verde de Agroecologia e Desenvolvimento Sustentável, 12(1), 161-166.

Fontenele, L. M. S., Azevedo, M. L. X., Cardoso Filho, F. d. C., Muratori, M. C. S., Sá, L. R. S. D., \& Pereira, M. M. G. (20 15). Qualidade microbiológica do alho (Allium sativum) produzido e comercializado em mercados públicos. Rev Inst Adolfo Lutz, 74(4), 420-425.

Caldas, F. F., Silva Filho, J. P., Rodrigues, C. A. R., \& da Silva, D. P. (2019). Atividade antimicrobiana do alho (Allium sativum L.) frente a bactéria causadora de infecção do trato urinário. Journal of Biotechnology and Biodiversity, 7(1), 217-224.

Hoog, G. S., Guarro, J., Gené, J., \& Figueras, M. J. (2000). Atlas of clinical fungi, (2a ed.), CBS, Spain. 1126p.

Lacaz, C. S., Porto, E., Martins, J. E. C., Heins-Vaccari, E. M., \& Melo, N. T. (2002). Tratado de Micologia médica; Prefácio: Bertrand Dupont. (9a ed.), Sarvier. $1104 \mathrm{p}$.

Leite, C. D., Maia, A. J., Botelho, R. V., Faria, C. M. D. R., \& Machado, D. (2012). Extrato de alho no controle in vitro e in vivo da antracnose da videira. Revista Brasileira de Plantas Medicinais, 14(3), 556-562.

Lubian, C.T, Teixeira, J.M, Lund, R.G, Nascente, P.S, \& Del Pino, F.A.B (2010). Atividade antifúngica do extrato aquoso de Arctium minus (Hill) Bernh. (Asteraceae) sobre espécies orais de Candida. Revista Brasileira de Plantas Medicinais, 12 (2), 157-162.

Oliveira, J. O., Santos, I. M. R., Silva, D. P., Calumby, R. J. N., Moreira, R. T. F., \& Araújo, M. A. S. (2020). Ocorrência de fungos na água e areia de praias urbanas. Diversitas Journal, 5 (4), 2779-2791.

Pereira, F. O., Lima, E. O., Figueiredo, K. R. L., Brito, L. L., \& Meira, A. S. (2010). Microbiota fúngica do solo e ar atmosférico na região da Borborema, estado da Paraíba, Brasil. Revista Brasileira de Análises Clínicas, 42 (2), 123-126.

Takahashi, J. P., Pelegrini, A., Pereira, C. Q. M, \& Souza, M. C. (2011). Levantamento de fungos queratinofílicos em solo de parques e praças públicas no município de São Bernardo do Campo. Revista de Biologia e Ciências da Terra, 11 (1), 47-53.

Santos, M. B., Santos, C. Y., Almeida, M. A., Santos, C. R. S., Sant'Anna, H. L. S., Santos, O. S. N., Silva, F., \& Martins, G. N. (2010). Efeito inibitório in vitro de extrato vegetal de Allium sativum sobre Aspergillus niger Tiegh. Revista Brasileira de Plantas Medicinais, 12(1), 13-17.

Sidrim, J. J. C., \& Rocha, M. F. G. (2010). Micologia Médica à luz de autores contemporâneos. (2a ed.), Guanabara Koogan.

Silva, S. B. D., Santos, A. N. D., \& Siqueira, L. D. P. (2020). Ação antimicrobiana e toxicidade do óleo essencial de melaleuca (Melaleuca alternifolia) e da alicina, sua utilização em formas farmacêuticas e possível associação para o tratamento de infecções dérmicas. Brasilian Journal of Development, 66(6), 3455534565 .

Sobral, N. V., Miranda,Z. D., \& Mascarenhas e Silva, F. (2018). Estratégia para a recuperação de informação científica sobre as doenças tropicais negligenciadas: análise comparativa da Scopus, Pubmed e Web of Science. Revista Cubana de Información en Ciencias de la Salud, 29 (1), 74-91.

Zaitz, C., Campbell, I., Marques, A. S., Ruiz, L. R. B., \& Souza, V. M. (2010). Compêndio de micologia médica. (2a ed.), Médica e Científica. 